\title{
Design and Implementation an Automated System for Analyzing Brushstrokes to Distinguish between Van Gogh and his Contemporaries by using Swarm Intelligent Method
}

\author{
Laheeb Mohammed Ibrahim \\ Software Engineering Dept. \\ Mosul University, Collage of Computer SC. \& Math, Mosul, Iraq
}

\begin{abstract}
In this paper, An Automated System for Distinguish Between Van Gogh and his Contemporaries by using Swarm Intelligent is designed. Swarm intelligence can be defined as the collective intelligence that emerges from a group of simple entities; these agents enter into interactions, sense and change their environment locally.

A system for Distinguish Between Van Gogh painting and his Contemporaries consists of three steps : processing step (In processing step, the digital paintings for Van Gogh and his contemporaries are processed automatically through many steps (Edge detection(Canny Edge detection and Ant colony optimization method), Edge linking (Hough transform method), Extract all the connected components from the image, Thinning and Resize image), feature extraction step based on histogram of oriented gradients (HOG), and recognition step using Artificial Fish Swarm Algorithm .

An Automated System for Distinguish Between Van Gogh and his Contemporaries by using Swarm Intelligent have been tested, and the recognized rate is $94.51 \%$.
\end{abstract}

\section{Keywords}

Swarm intelligence, feature extraction, Canny Edge detection method, Ant colony optimization method (ACO), Hough transform, Histogram of Oriented Gradients (HOG), Artificial Fish Swarm Algorithm.

\section{INTRODUCTION}

One important task for art historians is to find influences and connections between artists. By doing so, the conversation of art continues and new intuitions about art can be made. An artist might be inspired by one painting, a body of work, or even an entire style of art. Which artists influence each other? .Art historians are able to find which artists influence each other by examining the same descriptive attributes of art as space, texture, form, shape, color, tone, line, movement, unity, harmony, variety, balance, contrast, proportion, and pattern; besides brush strokes, subject matter, and other descriptive concepts. Similarities are noted and inferences are suggested [1][2].

Determining a painting's authenticity can be extremely challenging. Typically, art experts reach decisions after thorough consideration of many different types of evidence. Correspondence from the artist's lifetime and documents tracing the painting's history of ownership provide clues. Technical analyses of the pigments and other materials used and the method of their preparation [3].

It must be mentioned that determining influence is always a subjective decision. They will not know if an artist was ever

truly inspired by a work unless he or she has said, so for the sake of finding connections and progressing through movements of art, a general consensus is agreed upon if the argument is convincing enough.[4][5]. For example, (see Figure 1),illustrates a commonly cited comparison for studying influence, in the work of Van Gogh and Artist Yahya Zaki, and other Van Gogh Contemporaries where similarity is clear in composition, pose, and subject matter.

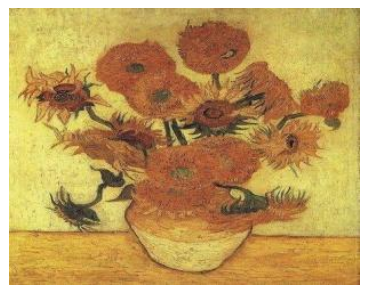

(a)
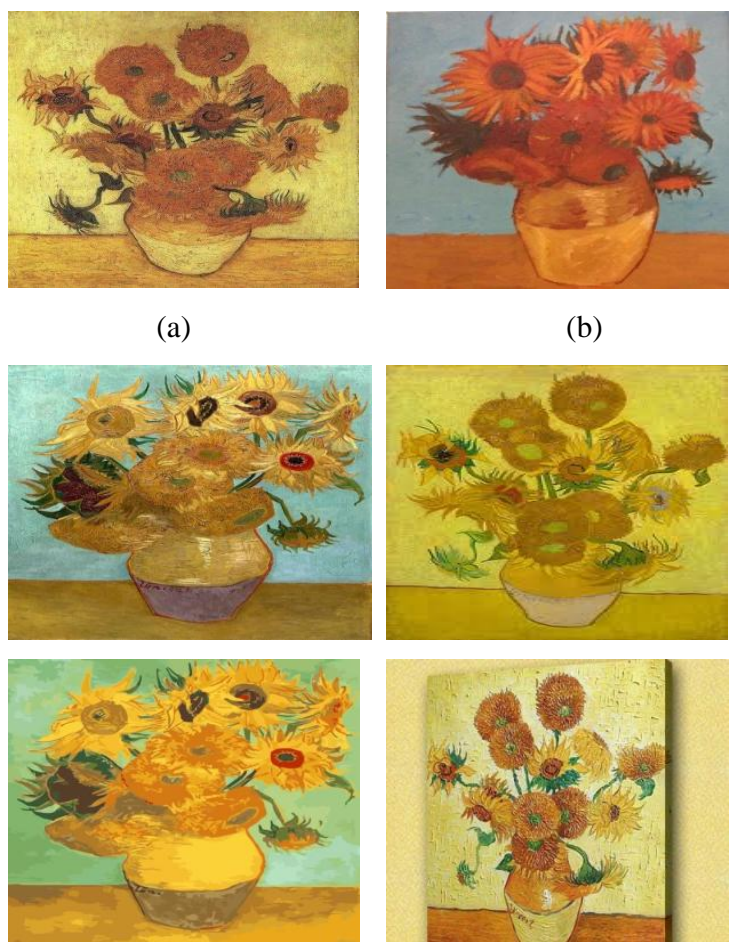

(b)
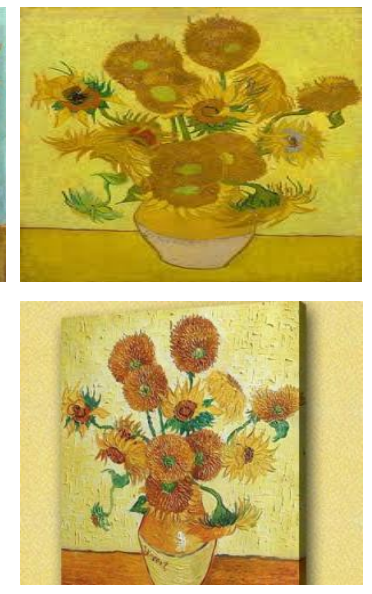

(c)

Fig. 1: Example of influence, in the work of Van Gogh and Artist Yahya Zaki, where similarity is clear in composition, pose, and subject matter. a : Van Gogh painting,b:Yahya Zaki painting,c:Van Gogh Contemporaries.

The authentication is based on connoisseurship and so relies upon the discerning eyes of a few experts who are steeped in the work and life of the artist in question. Other desiderata may include provenance which might be traced back to the artist's circle or his collectors and makes possible the comparison of the work's implicit biography with the histories of related works, or even a detailed analysis of any signature that may be present. 
In addition to the reliance on the human actor, quantitative methods can be brought to bear. X-ray analysis can reveal a painting beneath a painting that can shed light on its origins. Surface analysis of the painting materials is another approach, most famously applied in the investigation of the famous [24]. With the advent of digital technology, computational tools may be able to provide new insights and techniques into the art and science of art authentication. For example, a fractal analysis of Jackson Pollock's drip paintings has revealed interesting relations between the evolution of Pollock's aesthetic and the fractal dimension of his work. The analysis also raises the possibility of using fractal dimension to help authenticate Pollock. Various techniques from machine learning have been applied to the analysis and classification of craquelure . the crack lines that appear over time in a painting [6].

It has recently been proposed that computational tools from image analysis and machine learning can provide an additional source of analysis of questioned paintings. This assumes that an artist's brushwork is characterized by signature features (caused, e.g., by the artist's habitual physical movements) which might be found by machine learning methods and used as an additional piece of evidence to rule upon authenticity. Indeed, early attempts in this area have already found considerable success [7].

Although there has been some research on automated authentication of paintings, however, there is almost a few researches done on computer- based measuring and determining of influence between artists. Measuring influence is a very difficult task because of the broad criteria for what influence between artists can mean. As mentioned earlier, there are many different ways in which paintings can be described. Some of these descriptions can be translated to a computer [8].

The influence between artists need for an object-based representation of images. They should be able to describe the painting from a list of many different object classes, by having an object-based representation, the image is described in a high-level semantic as opposed to low-level features such as color and texture, which facilitates suggesting influences based on subject matter.

The benefit of the study of automated methods artistic influences about which artists and paintings may have similarities, is to keeps the conversation going for artists, also there are various application oriented motivations. With the increasing volumes of digitized art databases on the internet comes the daunting task of organization and retrieval of paintings. There are millions of paintings present on the internet. To manage properly the databases of these paintings, it becomes very essential to classify paintings into different categories. This classification structure can be utilized as an index and thus can improve the speed of retrieval process. Also it will be of great significance if they can infer new information about an unknown painting using already existing databases of paintings, and as a broader view can infer highlevel information like influences between painters. Time is also an essential factor in determining influence. An artist cannot influence another artist in the past. Therefore the linearity of paintings cuts down the possibilities of influence. Measuring influence is a very difficult task because of the broad criteria for what influence between artists can mean. There are many different ways in which paintings can be described. Some of these descriptions can be translated to a computer.
In this paper, an automated system is designed and implemented to use similarity measures to find influences and to recognized between Van Gogh painting and his Contemporaries based on swarm intelligent methods. The main contributions of this paper include Extracting individual brushstrokes automatically is a challenging problem partly due to the intermingling nature of brushstrokes in paintings and the low contrast in some painted areas . A Histogram of Oriented Gradients (HOG) is proposed to characterize brushstrokes, and Artificial Fish Swarm authentication method are proposed to recognized between Van Gogh and his Contemporaries

\section{RELATED WORK}

Babak Saleh, et. al in 2016 [8], exploring the problem of computer automated suggestion of influences between artists, a problem that was not addressed before in a general setting. They first present a comparative study of different classification methodologies for the task of fine- art style classification. A two-level comparative study is performed for this classification problem. The first level reviews the performance of discriminative vs. generative models, while the second level touches the features aspect of the paintings and compares semantic- level features vs. low-level and intermediate-level features present in the painting. Then, the investigate the question IWho influenced this artist?" by looking at his masterpieces and comparing them to others. They pose this interesting question as a knowledge discovery problem. For this purpose, they investigated several paintingsimilarity and artist-similarity measures. As a result, They provide a visualization of artists (Map of Artists) based on the similarity between their works.

Ahmed Elgammaly et. al., In 2015 [1], developed a computer algorithm that assesses the creativity of a painting given its context within art history. This paper proposes a novel computational framework for assessing the creativity of creative products, such as paintings, sculptures, poetry, etc. they use the most common definition of creativity, which emphasizes the originality of the product and its influential value. The proposed computational framework is based on constructing a network between creative products and using this network to infer about the originality and influence of its nodes. Through a series of transformations, they construct a Creativity Implication Network. They show that inference about creativity in this network reduces to a variant of network centrality problems which can be solved efficiently. They apply the proposed framework to the task of quantifying creativity of paintings (and sculptures). They experimented on two datasets with over $62 \mathrm{~K}$ paintings to illustrate the behavior of the proposed framework. They also propose a methodology for quantitatively validating the results of the proposed algorithm, which they call the "time machine experiment". Gustavo Carneiro in 2013 [9], introduced a new methodology for the problem of artistic image analysis, which among other tasks, involves the automatic identification of visual classes present in an art work. In this paper he advocate the idea that artistic image analysis must explore a graph that captures the network of artistic influences by computing the similarities in terms of appearance and manual annotation. One of the novelties of his methodology is the fact that the proposed formulation is a principled way of combining these two similarities in a single graph. Using this graph, he show that an efficient random walk algorithm based on an inverted label propagation formulation produces more accurate annotation and retrieval results compared to the following baseline algorithms: bag of visual words, label 
propagation, matrix completion, and structural learning. he also show that the proposed approach leads to a more efficient inference and training procedures. This experiment is run on a database containing 988 artistic images (with 49 visual classification problems divided into a multiclass problem with 27 classes and 48 binary problems), where he show the inference and training running times, and quantitative comparisons with respect to several retrieval and annotation performance measures.

Jia Li, et.al in 2012 [5], they compared van Gogh painting with his contemporaries by statistically analyzing a massive set of automatically extracted brushstrokes. A novel extraction method is developed by exploiting an integration of edge detection and clustering- based segmentation. Evidence substantiates that van Gogh's brushstrokes are strongly rhythmic. That is, regularly shaped brushstrokes are tightly arranged, creating a repetitive and patterned impression. They also found that the traits that distinguish van Gogh's paintings in different time periods of his development are all different from those distinguishing van Gogh from his peers. This study confirms that the combined brushwork features identified as special to van Gogh are consistently held throughout his French periods of production (1886-1890).

Ravneet Singh Arora, et. al in 2012 [10], presents a comparative study of different classification methodologies for the task of fine-art genre classification. 2-level comparative study is performed for this classification problem. 1st level reviews the performance of discriminative vs. generative models while 2 nd level touches the features aspect of the paintings and compares Semantic-level features vs low-level and intermediate level features present in the painting.

Ricardo S. Cabral, et al in 2011 [11], they presented a framework for estimating the ordering and date information of paintings and drawings. They formulate this problem as the embedding into a one dimension manifold, which aims to place paintings far or close to each other according to a measure of similarity. Their formulation can be seen as a manifold learning algorithm, albeit properly adapted to deal with existing questions in the art community. To solve this problem, they propose an approach based in Laplacian Eigenmaps and a convex optimization formulation. Both methods are able to incorporate art expertise as priors to the estimation, in the form of constraints. Types of information include exact or approximate dating and partial orderings. They explore the use of soft penalty terms to allow for constraint violation to account for the fact that prior knowledge may contain small errors. Their problem is tested within the scope of the PrintART project, which aims to assist art historians in tracing Portuguese Tile art_Azulejos_back to the engravings that inspired them. Ella Hendriks, et. al in 2007 [12], they process attributing paintings relies partly upon recognition of an artist's hand. Around the middle of the last century, Maurits M. van Dantzig (1903-1960) attempted to define the 'characteristic touch' of the painter, Vincent van Gogh (1853-1890). His broader aim was to develop a flexible yet precise method to measure the features of both spontaneity and inhibition, evident in the style of brushwork for example. The underlying idea that these qualities can be used to separate a genuine work from a second-rate copy or forgery still plays a key role in attribution studies today. A recent initiative explores the potential of advanced computer image analysis techniques to help identify and quantify these properties at the scale of brushwork. This paper describes the basic principles of the method used, involving statistical analysis of different size wavelets present in digital images of paintings by Van Gogh or other artists

Igor Berezhnoy ,et al. in 2007 [13], Traditionally, the analysis of visual arts is performed by human art experts only. The availability of advanced artificial intelligence techniques makes it possible to support art experts in their judgment of visual art. In this paper image-analysis techniques are applied to measure the complementary colors in the oeuvre of Vincent van Gogh. It is commonly acknowledged that, especially in his French period, Van Gogh started employed complementary colors to emphasize contours of objects or parts of scenes. They propose a method to measure complementary-color usage in a painting by combing an opponent-color space representation with Gabor filtering. Using this method, the analysis of a dataset of 617 digitized oil-on-canvas paintings confirms art expert's knowledge about the global pattern of complementary-color usage in Van Gogh's paintings. In addition, it provides an objective and quantifiable way to support the analysis of colors in individual paintings. Our results show that art experts can be supported by artificial-intelligence technique.

Siwei Lyu, et. al in 2004 [6], described a computational technique for authenticating works of art, specifically, paintings and drawings, from high resolution digital scans of the original works. This approach builds a statistical model of an artist from the scans of a set of authenticated works, against which new works are then compared. The statistical model consists of first and higher-order wavelet statistics. They show preliminary results from their analysis of thirteen drawings that have at various times been attributed to Pieter Bruegel the Elder, which confirm expert authentications. They also apply these techniques to the problem of determining the number of artists that may have contributed to a painting attributed to Perugino and again achieve an analysis agreeing with expert opinion.

After study researches in the field of authentication of paintings and especially in the area for distinguish Plates and brushstrokes Between Van Gogh and his Contemporaries artist, the researcher found that there are problems specializes in the process of segmentation brushstrokes appropriate for feature extraction and in the process of recognition operation. And also all researchers did not use in their researches swarm intelligent algorithms in the process of segmentation brushstrokes and recognition, for this reason, in this research, an automated system based on swarm intelligent algorithms to segmentation brushstrokes, feature extraction and to recognized brushstrokes Between Van Gogh and his Contemporaries artist is designed.

\section{AN AUTOMATED DISTINGUISH BRUSHSTROKES BETWEEN VAN GOGH AND HIS CONTEMPORARIES SYSTEM}

To design an automated system to distinguish brushstrokes between Van Gogh from his contemporaries, it must first execute processing step on digital paintings for Van Gogh and his contemporaries, and then extract features from these processed digital paintings, after features are extracted the recognition step is done to distinguish brushstrokes between to brushstrokes Van Gogh and his contemporaries, (see Figure 2). 


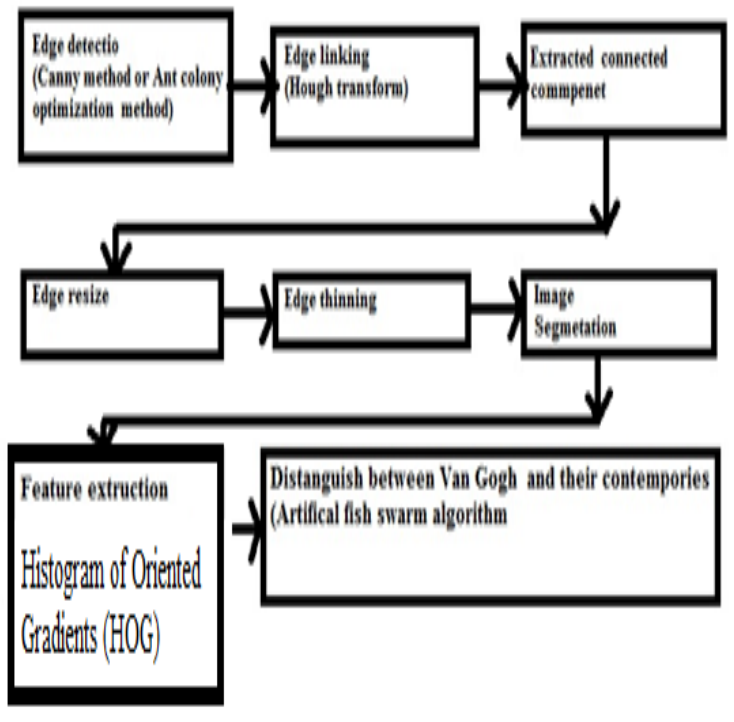

Fig. 2: An automated distinguish brushstrokes between van Gogh and his contemporaries system

The following steps explain how to distinguish brushstrokes between Van Gogh from his contemporaries.

\subsection{Processing Steps}

In processing steps, the digital paintings for Van Gogh and his contemporaries are processed automatically through many steps (Edge detection, Edge linking, Extract all the connected components from the image, Thinning and Resize image).

\section{1- Proposed Edge Detection Method}

Edge information is one of the most important information in an image, which can describe the target outline, the relative position within the target area, and other important information. Edge detection is one of the most important process in image processing, and the detection results will directly affect the image analysis. In this paper two methods are used to detected edge (Canny Edge detection and Ant colony optimization method).

\section{- Canny Edge detection method}

The Canny edge detector is an edge detection operator that uses a multi-stage algorithm to detect a wide range of edges in images. It was developed by John F. Canny in 1986. Canny also produced a computational theory of edge detection explaining why the technique works. Canny edge detection is a technique to extract useful structural information from different vision objects and dramatically reduce the amount of data to be processed. It has been widely applied in various computer vision systems. Canny has found that the requirements for the application of edge detection on diverse vision systems are relatively similar. Thus, an edge detection solution to address these requirements can be implemented in a wide range of situations. Among the edge detection methods developed so far, canny edge detection algorithm is one of the most strictly defined methods that provides good and reliable detection. Owing to its optimality to meet with the three criteria for edge detection and the simplicity of process for implementation, it became one of the most popular algorithms for edge detection. The steps in the canny edge detector algorithm are as follows[14]:

\section{Algorithm .1 Canny Edge Detection}

1. Smooth the image with a Gaussian filter.

2. 2.Compute the gradient magnitude and orientation using finite-difference approximations for the partial derivatives.

3. Non-maximal suppression. Edges will occur at points the where the gradient is at a maximum. Therefore, all points not at a maximum should be suppressed. In order to do this, the magnitude and direction of the gradient is computed at each pixel. Then for each pixel check if the magnitude of the gradient is greater at one pixel's distance away in either the positive or the negative direction perpendicular to the gradient. If the pixel is not greater than both, suppress it.

4. Edge Thresholding. The method of thresholding used by the Canny Edge Detector is referred to as "hysteresis". It makes use of both a high threshold and a low threshold. If a pixel has a value above the high threshold, it is set as an edge pixel. If a pixel has a value above the low threshold and is the neighbor of an edge pixel, it is set as an edge pixel as well. If a pixel has a value above the low threshold but is not the neighbor of an edge pixel, it is not set as an edge pixel. If a pixel has a value below the low threshold, it is never set as an edge pixel. (Algorithm based on description given in [15]).

The proposed Canny edge detection method was implemented using Matlab Ver. 7.11 on $(1000 \times 100$ pixels $)$ image. Figure 3, show the experiment result for canny detection method, a :Canny Edge detection operation on Van Gogh painting, b: represent Canny Edge detection operation on Artist Yahya Zaki painting.

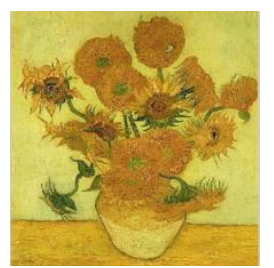

Original image

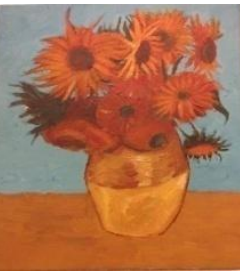

Original image

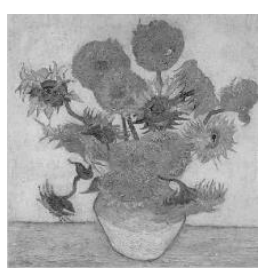

gray converted Image

(a)

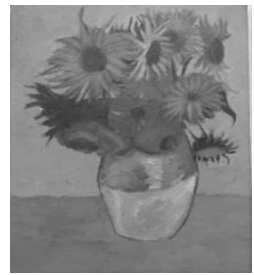

gray converted Image

(b)

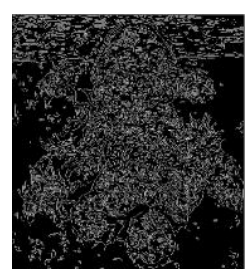

Canny detection result

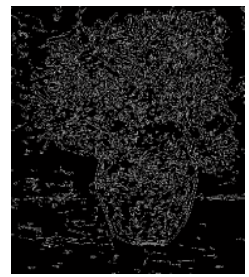

Canny detection result
Fig 3: A Canny Edge detection operation, a : Canny Edge detection operation on Van Gogh painting b : Canny Edge detection operation on Artist Yahya Zaki painting.

- Ant colony optimization method (ACO)

This section provides a theoretical discussion on the ant colony optimization metaheuristic, ant colony system, and proposed ACO-based image edge detection.

ACO is used to evolve the edge information existing in the image, since it is critical to understand the image's content. The ACO algorithm uses artificial ants as a simple 
computational agent. It is initialized with $\mathrm{N}$ ants that occupy "random" pixels within the image, here the image pixels are equivalent to states in the search environment. The aim of the ants is to locate and plot the boundaries within the image. To achieve this heuristic information is introduced. With each move to a new pixel every ant deposits an amount of pheromone, where the amount deposited may be a function of, e.g. change in image gradient, and pheromone evaporation occurs at a fixed rate per iteration. The transition rule is then a function of heuristic information and pheromone map [16][17].

ACO is a probabilistic technique for finding optimal paths in fully connected graphs through a guided search, by making use of the pheromone information. This technique can be used to solve any computational problem that can be reduced to finding good paths on a weighted graph. In an ACO algorithm, ants move through a search space, the graph, which consists of nodes and edges. The movement of the ants is probabilistically dictated by the transition probabilities. The transition probability reflects the likelihood that an ant will move from a given node to another. This value is influenced by the heuristic information and the pheromone information. The heuristic information is solely dependent on the instance of the problem. Pheromone values are used and updated during the search[16][17]

Artificial ants iterates tour construction loop which is biased with the artificial pheromone trails and the heuristic information. The main mechanism at work in ACO is the discovery of good tours is the positive feedback done through the pheromone update by the ants. The shorter the ant's tour, the more amount of pheromone is deposited by ants. This forces the ants to select the same arcs in the subsequent iterations of the algorithm. The occurrence of arcs with high pheromone values are further reinforced by the mechanism of pheromone evaporation that avoids an unlimited amount of pheromone and decrease the pheromone content from the arcs that rarely receive additional pheromone [18]. Algorithm below describe how ACO is work :

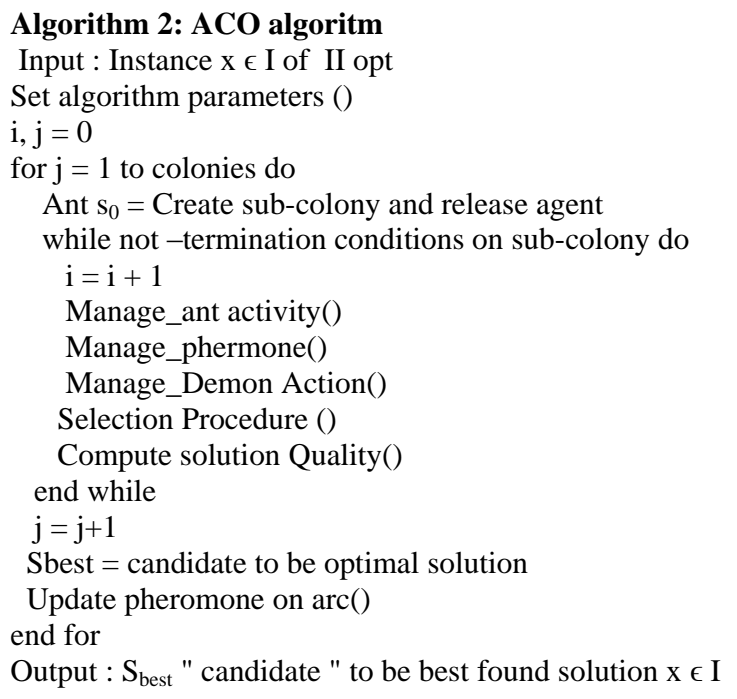

As in [16] [17] [18], number of ants move on a 2-D image, stepping from one pixel to another to construct a pheromone matrix, which determine the edge information for each pixel location in the image to extract the edges of the image. The movement of the ants is directed by the local variation of the image's intensity values. Image Edge detection process has the following steps: first is the initialization process. After this pheromone matrix is constructed by the ACO when it further runs for $\mathrm{N}$ no. of iterations. Iterative process consists of construction process and update process. The last is decision process by which edge is determined.

\section{Initialization process}

In this process for an image $\mathrm{I}$ of size $\mathrm{M} \times \mathrm{N}$ is taken as input which works as a solution space for the artificial ants. The $\mathrm{K}$ numbers of ants are randomly moved over the whole image such that the every pixel of the image is viewed as a node. The constant is assigned to each, which is the initial value of every component of the pheromone matrix.

2. Construction Process

In the nth step of construction, one ant being randomly selected from $\mathrm{K}$ total ants and this ant will move over the image for $\mathrm{L}$ steps. This ant will move from the $(1, \mathrm{~m})$ node to $(i, j)$ node which is its neighbouring node or pixel, is specified by the transition probability.

\section{Update Process}

The pheromone matrix is updated in the update process after the two update operations. The first update is accomplished after the movement of each ant in each construction-step. Each building block of pheromone matrix is modified.

\section{Decision process}

The solution is based on the values in the final pheromone matrix. The literature applies a threshold technique, also known as the Otsu threshold technique. or by the method developed to reduce the resulting grey scale image to a binary image with only two possible values for each pixel. This is done to be able to classify each pixel as either an edge or a non-edge. Though, when it comes to analyzing the work carried out by the ant collective in image edge detection, a result showing various degrees in intensity values is just as good as a black and white declaration. Hence, in an ant's image edge detection, the solution is a direct result of the values in the final pheromone matrix In this step, a binary decision is made at each pixel location to determine whether it is edge or not. The decision is made by applying a threshold $\tau$ on the final pheromone matrix. Here the threshold value $\tau$ chosen to be adaptively computed.

5. Visualize Process

In this step, different values of the $\mathrm{Si}(\psi)$ parameter are applied to the above algorithm. Smaller the value of the phi parameter more edges the algorithm detects in the image. As go on decreasing the value of the phi parameter, output of the given image becomes clearer but it should not be zero.

The proposed Ant colony optimization (ACO) method was implemented using MatlabVer. 7.11 on $(1000 \times 100$ pixels $)$ image. Figure 4 show the experiment result for ACO detection method, a :ACO Edge detection operation on Van Gogh painting, b: represent ACO Edge detection operation on Artist Yahya Zaki painting.

\subsection{Brushstrokes Image Segmentation Step}

The edge linking algorithm is applied in this step to remove short noisy edges and trace every legitimate edge and record the coordinates of the pixels on the edge in the tracing order. The Hough transform method is used to find connected sets of edge pixels, without having to explicitly first identify which are or are not edges is to trace from pixel to pixel through possible edge points, considering as you go the context along the path. The Hough transform is a technique which can be used to isolate features of a particular shape within an image. Hough transform is most commonly used for the detection of regular curves such as lines, circles, ellipses, etc. The main 
advantage of the Hough transform technique is that it is tolerant of gaps in feature boundary descriptions and is relatively unaffected by image noise [19].

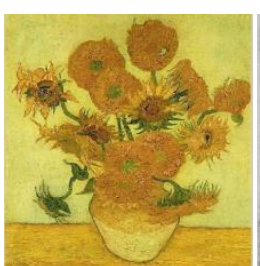

Original image

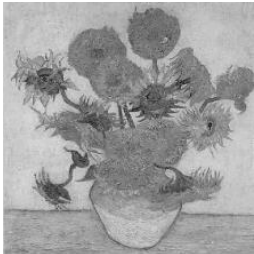

gray converted Image

(a)

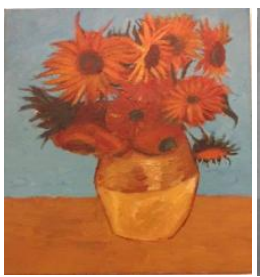

Original image

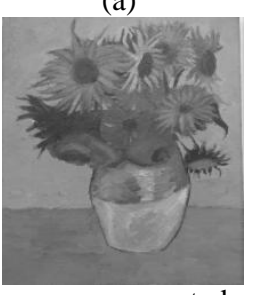

gray converted Image

(b)

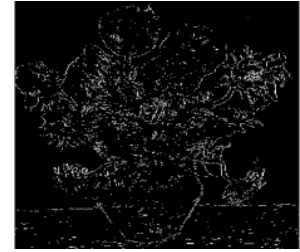

ACO detection result

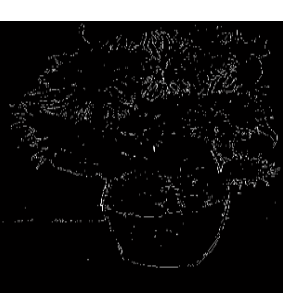

$\mathrm{ACO}$ detection result
Fig 4: A Canny Edge detection operation, a : Canny Edge detection operation on Van Gogh painting b : Canny Edge detection operation on Artist Yahya Zaki painting.

Algorithm 3 : Hough transform [20] .

1. Quantize the parameter space $(a, b)$, that is, divide it into cells.

2. This quantized space is often referred to as the accumulator cells.

3. In the figure in the next slide $\mathrm{a}_{\min }$ is the minimal value of a etc.

4. Count the number of times a line intersects a given cell.

- For each point $(\mathrm{x}, \mathrm{y})$ with value 1 in the binary image, find the values of $(a, b)$ in the range $\left[\left[\mathrm{a}_{\min }, \mathrm{a}_{\max }\right],\left[\mathrm{b}_{\min }, \mathrm{b}_{\max }\right]\right]$ defining the line corresponding to this point.

- Increase the value of the accumulator for these [a',b'] point.

- Then proceed with the next point in the image.

5. Cells receiving a minimum number of "votes" are assumed to correspond to lines in $(\mathrm{x}, \mathrm{y})$ space.

- Lines can be found as peaks in this accumulator space.

After Edge linking step, A brushstroke fully enclosed by an edge is spatially isolated from other non-edge pixels and forms a connected component, as in [5 ][21], extract all the connected components from the image by setting the edge pixels as foreground, and the non- edge pixels as background. After extracted a connected component from the previous step, The Resize operation on the image is executed, a brushstroke must be in size (256 x 265 pixel). As in [22]

Skeletons for candidate brushstrokes are obtained by the thinning operation. Thinning is a morphological operation, is commonly used to tidy up the output of edge detectors by reducing all lines to single pixel thickness. See [23]

The next step is to perform image segmentation by clustering all the pixel-level features in the image. The clustering algorithm applies k-means multiple times with a gradually decreasing threshold for the average within-cluster distance.
As in [5], Figure 5 illustrates the process using real candidate brushstroke segments and their skeletons

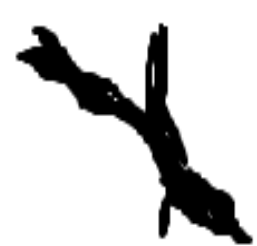

(a)

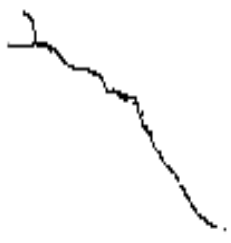

(b)
Fig. 5: The process using real candidate brushstroke segments and their skeletons (a) brushstrokes extracted image (b) brushstrokes extracted image after processing steps

\subsection{Feature Extraction}

The result from Brushstrokes image processing step is used as input to histogram of oriented gradients (HOG) feature extraction. The histogram of oriented gradients (HOG) is a feature descriptor used in computer vision and image processing for the purpose of feature extraction and object detection. The technique counts occurrences of gradient orientation in localized portions of an image. This method is similar to that of edge orientation histograms, scale-invariant feature transform descriptors, and shape contexts, but differs in that it is computed on a dense grid of uniformly spaced cells and uses overlapping local contrast normalization for improved accuracy.

The essential thought behind the histogram of oriented gradients descriptor is that local object appearance and shape within an image can be described by the distribution of intensity gradients or edge directions. The image is divided into small connected regions called cells, and for the pixels within each cell, a histogram of gradient directions is compiled. The descriptor is the concatenation of these histograms. For improved accuracy, the local histograms can be contrast-normalized by calculating a measure of the intensity across a larger region of the image, called a block, and then using this value to normalize all cells within the block. This normalization results in better invariance to changes in illumination and shadowing [24].

In this section, the details of calculating the HOG feature extraction is described, The HOG feature extraction used in this paper work as in [24] as follow

Step 1 : Calculate the Gradient Images : As mentioned earlier HOG feature descriptor used for feature extraction is calculated on a $256 \times 256$ patch of an image, see Figure 5 (b), To calculate a HOG descriptor, calculate the gradient images need to first calculate the horizontal and vertical gradients; after all, want to calculate the histogram of gradients. This is easily achieved by filtering the image with the following kernels.

\begin{tabular}{|l|l|l|}
\hline-1 & 0 & 1 \\
\hline
\end{tabular}

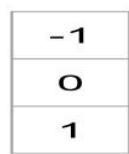

$$
\begin{aligned}
& g=\sqrt{g_{x}^{2}+g_{y}^{2}} \\
& \theta=\arctan \frac{g_{y}}{g_{x}}
\end{aligned}
$$


Step 2 : Calculate Histogram of Gradients in $8 \times 8$ cells : In this step, the image is divided into $8 \times 8$ cells and a histogram of gradients is calculated for each $8 \times 8$ cells. The gradient of this patch contains 2 values ( magnitude and direction ) per pixel which adds up to $8 \times 8 \times 2=128$ numbers. these 128 numbers are represented using a 9- bin histogram which can be stored as an array of 9 numbers, the histogram is essentially a vector ( or an array ) of 9 bins ( numbers ) corresponding to angles $0,20,40,60 \ldots 160$. Figure (6) represent one $8 \times 8$ patch in the image and see how the gradients look.

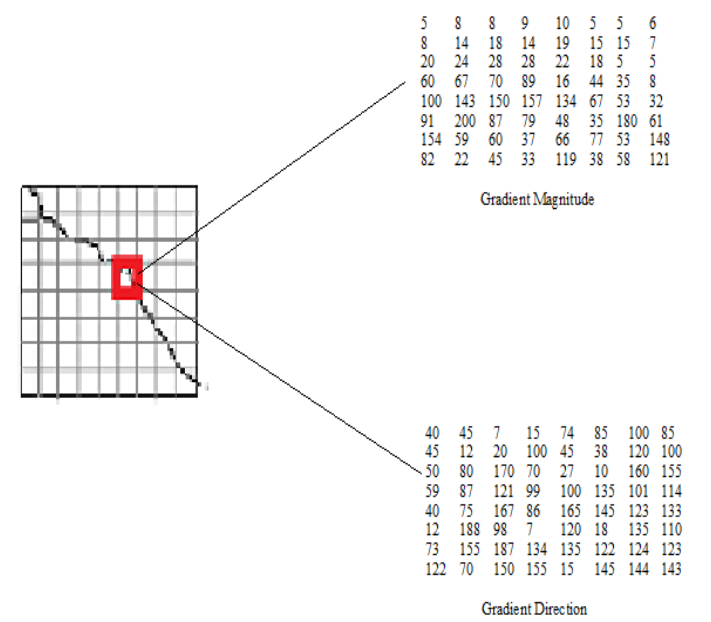

Fig. 6 : one $8 \times 8$ patch in the image and the gradients

Step 3: Create a histogram of gradients in these $8 \times 8$ cells: The histogram contains 9 bins corresponding to angles 0,20 , $40 \ldots 160$. The operation is done on magnitude and direction of the gradient of the same $8 \times 8$ patch as in figure 6 . A bin is selected based on the direction, and the vote (the value that goes into the $b$ in ) is selected based on the magnitude. (See figure 7)

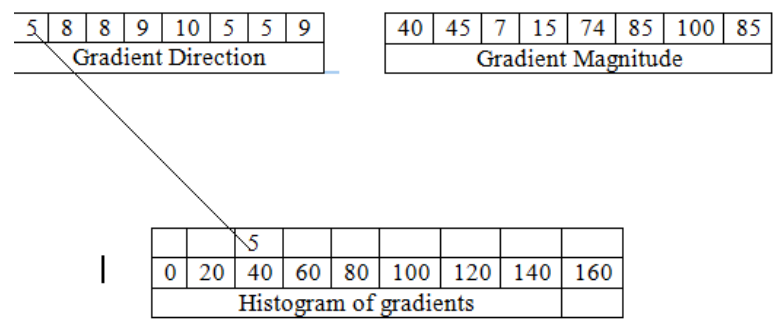

Fig. 7 : A bin selected based on the direction, and the vote selected based on the magnitude.

As in [24], If the angle is greater than 160 degrees, it is between 160 and 180, and we know the angle wraps around making 0 and 180 equivalent. the pixel with angle 165 degrees contributes proportionally to the 0 degree bin and the 160 degree bin.

The contributions of all the pixels in the $8 \times 8$ cells are added up to create the 9-bin histogram. For the patch above, it looks like figure 8 .

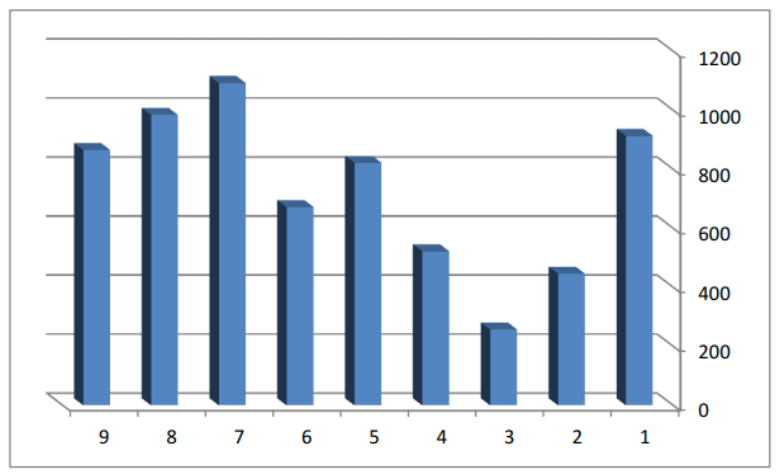

Fig. 8: The 9-bin histogram

Step 4 : Calculate the HOG feature vector : To calculate the final feature vector for the entire image patch, the $9 \times 1$ vectors are concatenated into one giant vector. The size of vector is calculated as for each $8 \times 8$ blocks there are 4 horizontal and 8 vertical position making a total $4 \times 8=32$ position, each $8 \times 8$ block is represented by $9 \times 1$ vector when it concatenate all into one gain vector we obtained $9 \times 32=$ 288 dimensional vector.

1. How many positions of the $16 \times 16$ blocks do we have ? There are 7 horizontal and 15 vertical positions making a total of $7 \times 15=105$ positions.

2. Each $16 \times 16$ block is represented by a $36 \times 1$ vector. So when we concatenate them all into one gaint vector we obtain a $36 \times 105=3780$ dimensional vector.

\subsection{Brushstrokes Recognition Between Van Gogh and his Contemporaries}

In this paper the Artificial Fish Swarm Algorithm was implemented in Matlab 7.11 to Distinguish Between Van Gogh and his Contemporaries.

The artificial fish swarm algorithm (AFSA) is one of the stateof-the-art swarm intelligence approaches, which was proposed by $\mathrm{Li}$ Xiaolei in 2002. It is inspired by the autonomous collective movement of artificial fishes (AFs) and their various social behaviors. Its characteristics of global search, quick convergence rate, and efficient search are based on modern elicitation methods. After AFSA appeared, it offered new ideas to solve the optimization problems in signal processing, neural network classifiers, data mining and clustering, multi-objective optimization and PID controller parameters optimization, pattern recognition ... etc[25].

As we know in water world, fishes can discover areas that have more foods, which is done with individual or swarm search by fishes. In relation to this characteristic, artificial fish (AF) model is represented by prey, free move, swarm and follow behaviors. AFs search the problem space by those behaviors. The location, in which AF lives, considerably is solution space and other AF's sphere. Objective function is Food consistence degree in water area is AFSA. Lastly, AFs attain to a point which its food consistence degree is maxima also referred as global optimum [25][26][27].

Supposed the state vector of artificial fish swarm is $\mathrm{X}=(\mathrm{x} 1$, $\mathrm{x} 2, \mathrm{x} 3, \ldots \mathrm{xn})$, where $\mathrm{x} 1, \mathrm{x} 2, \mathrm{x} 3, \ldots \mathrm{xn}$ is status of the fish. Visual is the visual distance, the artificial fish occurs only in the inner radius of the circle to the length of the field of vision various acHts. The food concentration in this position of fish is expressed as $y=f(x)$, Where $y$ is the objective function 
value. The distance between the artificial fish is $\mathrm{dij}=\| \mathrm{Xi}$ $\mathrm{Xj} \|$, $\mathrm{i}$ and $\mathrm{j}$ is a random fish. means the maximum step size of artificial fish. $\delta$ is the degree of congestion factor [27] [28][29].

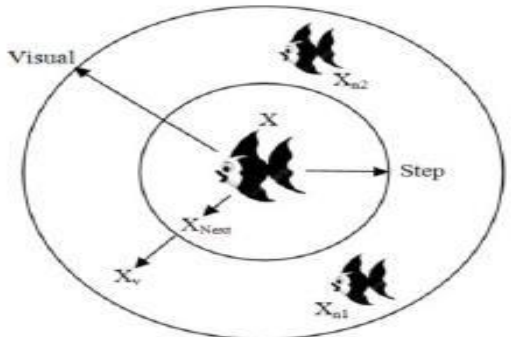

Fig. 9: Vision Concept of artificial Fish

Supposed $X_{v}$ is the visual position at some moment. $X_{\text {next }}$ is the new position. Than the movement process is represented as:

$X_{v}=X_{i}+$ Visual $x$ rand ()$, i \epsilon[0, n]$

$\mathrm{X}_{\text {next }}=\mathrm{X}+\left(\left(\mathrm{X}_{\mathrm{v}}-\mathrm{X}\right) /\left(\left\|\mathrm{X}_{\mathrm{v}}-\mathrm{X}\right\|\right)\right) \mathrm{x}$ step $\mathrm{x}$ rand ()

Where rand () produces random numbers between 0 and 1 .

The basic behaviors of artificial fish are defined as follows [27][30].

\section{Prey behavior}

This is a basic biological behavior that tends to the food. Supposed the state of artificial fish is $X_{i}$, Select a state $X_{j}$ within its sensing range randomly. If $X_{j}$ superior to $X_{i}$, then move to $X_{j}$; on the contrary, selected randomly state $X_{j}$ and determine whether to meet the forward conditions, repeated several time, if still not satisfied forward conditions, then move one step randomly.

$\mathrm{X}_{\mathrm{j}}=\mathrm{X}_{\mathrm{i}}+$ Visual $\times \operatorname{rand}()$

If $Y_{i}<Y_{j}$, it goes forward a step in this direction

$\mathrm{X}_{\mathrm{i}}^{\mathrm{t}+1}=\mathrm{X}_{\mathrm{i}}^{\mathrm{t}}+\left(\left(\mathrm{X}_{\mathrm{j}}-\mathrm{X}_{\mathrm{i}}^{\mathrm{t}}\right) /\left(\left\|\mathrm{X}_{\mathrm{j}}-\mathrm{X}_{\mathrm{i}}^{\mathrm{t}}\right\|\right)\right) \mathrm{x}$ step $\mathrm{x}$ rand ()

\section{Swarm Behavior}

Supposed the current state of artificial fish is $X_{i}\left(d_{i, j}<\right.$ Visual $)$, number of artificial fish is $\mathrm{n}_{\mathrm{f}}$, if $\mathrm{n}_{\mathrm{f}}<\delta$ indicates that the partners have more food and less crowded, if $Y_{c}$ better than $Y_{i}$ ,then go forward toward the center of the direction of the partnership, otherwise prey behavior.

$\mathrm{X}_{\mathrm{i}}^{\mathrm{t}+1}=\mathrm{X}_{\mathrm{i}}^{\mathrm{t}}+\left(\left(\mathrm{X}_{\mathrm{c}}-\mathrm{X}_{\mathrm{i}}^{\mathrm{t}}\right) /\left(\left\|\mathrm{X}_{\mathrm{c}}-\mathrm{X}_{\mathrm{i}}^{\mathrm{t}}\right\|\right)\right) \mathrm{x}$ step $\mathrm{x}$ rand ()

\section{Follow Behavior}

Supposed the state of artificial fish is $X_{i}$, explore its optimal state $\mathrm{X}$ max from Visual neighbors, the number of partners of $\mathrm{X}_{\max }$ is $\mathrm{n} \mathrm{f}$, If $\mathrm{n}_{\mathrm{f}}<\delta$ indicates that near distance have more food and not too crowded, further move to the front of $\mathrm{X}$ max position; otherwise perform foraging behavior.

$\mathrm{X}_{\mathrm{i}}^{\mathrm{t}+1}=\mathrm{X}_{\mathrm{i}}^{\mathrm{t}}+\left(\left(\mathrm{X}_{\mathrm{j}}-\mathrm{X}_{\mathrm{i}}^{\mathrm{t}}\right) /\left(\left\|\mathrm{X}_{\mathrm{j}}-\mathrm{X}_{\mathrm{i}}^{\mathrm{t}}\right\|\right)\right) \mathrm{x}$ step $\mathrm{x}$ rand ()

The artificial fish swarm algorithm is shown as:

\section{Algorithm 4: Artificial fish swarm algorithm}

1. Initialize the parameters of artificial fish, such as Step ,Visual , the number of exploratory try_number, maximum number of iterations, and randomly generated $\mathrm{n}$ fishes;

2. Set bulletin board to record the current status of each fish, and select the optimal value recorded;

3. Implementation of prey behavior, swarm behavior and follow behavior;

4. Optimal value in bulletin board is updated;

5. If the termination condition is satisfied, output the result; otherwise return to step 2.
The following steps specific how to distinguish between Van Gogh and his contemporaries by using AFSA

1. 1. Initialize AFSA parameters, artificial fish swarm size $(\mathrm{N}=160)$, Number of maximum iterations $=160$, the visual field of artificial fish (Visual $=3$ ) and the congestion factor $(\delta=1.6)$.

2. Set the initial iteration number $=0$, generate $(\mathrm{N}=$ 160) artificial fish individuals in the feasible domain of control variable and form initial fish swarm.

3. Set bulletin board to record the current status of each fish, and select the optimal value recorded by Calculate the food concentration (FC) of the current position of every fish individual in the initial fish swarm. Compare their FCs; record the maximum FC in the bulletin board and assign this fish to the bulletin board. Log of Euclidean Distance (LED)to be used in objective evaluation (food concentration (FC)). Where

$$
\mathrm{LED}=\sqrt{\sum_{i=1}^{n}(a(i)-b(i))^{2}}
$$

4. Every artificial fish simulates and implements follow and swarm respectively. The artificial fish with bigger FC implements the behavior in practice and the default behavior is forage.

5. After every artificial fish acts once, compare its FC with that on the bulletin board and if it is better than that on the bulletin board, replace it.

6. End condition judgment: judge whether num has reached the set-top maximum iterations number. If it is, output the optimal registration parameter set; otherwise, make number+=1 and turn to Step (4).

\section{EXPERIMENT RESULT}

The experiments is performed on 20 painting to compare van Gogh's paintings with his contemporaries using the ten paintings, 5 of which, referred to as van Gogh's, are by van Gogh and the other 15 referred to as non- van Gogh's, (3 Contemporaries painting for each Van Gogh painting), see figure 1 and figure 10 .
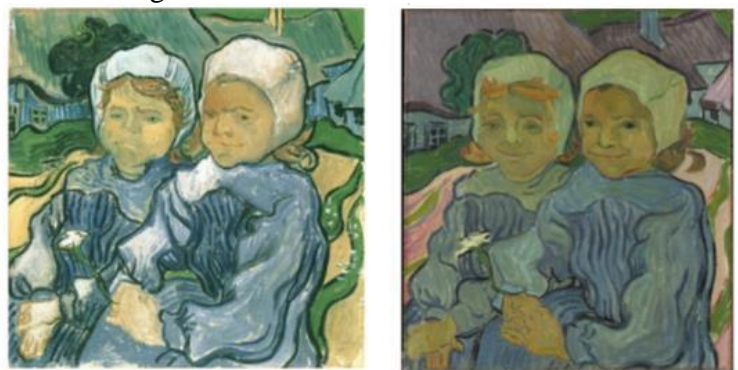

Fig. 10: a : van Gogh's painting b: non- van Gogh's painting

All 20 paintings are preprocessed, extracted features before recognized as van Gogh's non- van Gogh's painting, results are displayed in table 1

Table 1: Recognition rate of an automated distinguish brushstrokes between Van Gogh and his contemporaries system

\begin{tabular}{|c|c|}
\hline Edge Detection & Recognition Rate \\
\hline Canny Edge Detection & $91.14 \%$ \\
\hline $\begin{array}{l}\text { Ant } \\
\text { optimization } \text { method }\end{array}$ & $94.51 \%$ \\
\hline
\end{tabular}


On comparing Canny Edge detection approach with Ant colony optimization method approach for Edge detection, it is found that:. The average recognition rate of Ant colony optimization method is better than that of Canny Edge detection. Therefore, it can be said that the effectiveness of Ant colony optimization method in finding the optimal edge detection compared to Canny Edge detection compensates its computational inefficiency.

\section{CONCLUSION}

In this paper, an automated distinguish brushstrokes between Van Gogh and his contemporaries system was performed by applied Artificial Fish Swarm Algorithm as recognition algorithm. It was found out that the Artificial Fish Swarm Algorithm can be integrated into evolutionary computational algorithms to provide a better for distinguish brushstrokes between Van Gogh and his contemporaries. Finally, it is believed that the two edge detection (Canny Edge detection and Ant colony optimization method ) may be useful to edge detection for the development of distinguish brushstrokes between Van Gogh and his contemporaries

For future work, it can used anther swarm algorithms to segmented brushstrokes of Van Gogh and to distinguish brushstrokes between Van Gogh and his contemporaries.

\section{REFERENCES}

[1] Ahmed E., Babak S., 2015, Ahmed E., Babak S.,2015, "Quantifying Creativity in Art Networks", the sixth International Conference on Computational Creativity (ICCC), June 29- July 2nd 2015, Park City, Utah, USA.

[2] Babak S., Kanako A. R. Singh A., Ahmed E., 2016, " Toward Automated Discovery ofArtistic Influence, springer, April 2016, Volume 75, Issue 7, pp 3565-3591.

[3] El-bayoumy M.A. A., Rashad M. Z., Elsoud M. A., Eldosuky M. A., 2013, " Fast Artificial Fish Swarm Algorithm", International Journal of Information Science and Intelligent System, Vol. 2, No.4, 2013

[4] Fichner-Rathus L. , 2008. "Foundations of Art and Design". Clark Baxter.

[5] sensors : Gao Y, , Wang T, Sun Y.,2015, " A novel artificial fish swarm algorithm for recalibration of fiber optic gyroscope error parameters.", Sensors (Basel). 5;15(5):10547-68. ISSN 1424-8220 www.mdpi.com/journal/sensors

[6] G"ung"or P.n, Sina J., Andrei B., Shannon H., Ingrid D., 2009, "Detection Of Forgery In Paintings Using Supervised Learning", Image Processing (ICIP), 2009 16th IEEE International Conference on 7-10 Nov. 2009, Date Added to IEEE Xplore: 17 February 2010

[7] Gupta Ch. , Gupta S. ,2014, " Edge Detection of an Image based on Ant ColonyOptimization Technique", antInternational Journal of Science and Research (IJSR), India Online ISSN: 2319- 7064, Volume 2 Issue 6, www.ijsr.net

[8] Gustavo C., 2011, " Graph-based Methods for the Automatic Annotation and Retrieval of Art Prints", ICMR'11, April 17-20, Trento, Italy, Copyright c2011 ACM 978-1-4503-0336.
[9] Gustavo C., 2013, "Artistic Image Analysis using Graphbased Learning Approaches", IEEE Trans Image Process. 2013 Aug;22(8):3168-78.

[10] Hamid A. A., Majida A.A., 2015, " Object Recognition Using Artificial Fish Swarm Algorithm on Fourier Descriptors" ,American Journal of Engineering, Technology and Society, 2(5): 105- 110 Published online September 18 (http://www.openscienceonline.com/journal/ajets)

[11] Hendriks, E. , Shannon H.s, 2007, " Van Gogh's brushstrokes: marks of authenticity?", Chapter in book: Art, Conservation and Authenticities; Material, Concept, Context, Publisher: Archetype, Editors: Erma Hermens, Tina Fiske, pp.143-152 https://www.google.iq/?gfe_rd=cr\&ei=n7ApWOHLNqiz 8weqlq_4Bw\#q=Van+Gogh $\%$ E2\% $80 \%$

99s+brushstrokes:+marks+of+authenticity $\% 3 \mathrm{~F}+\mathrm{Ella}+\mathrm{He}$ ndriks*+and+Shannon+Hughes

[12] Igor Berezhnoy Eric Postma Jaap van den Herik, 2007, "Digital analysis of Van Gogh's complementary colours", Pattern Recognition, Volume 28 Issue 6, April, 2007,Pages703-709, Elsevier Science Inc. New York, NY, USA

[13] Jagadeesan R., 2013, "An artificial fish swarm optimized fuzzy mri image segmentation approach for improving identification of brain tumour" , International Journal on Computer Science and Engineering (IJCSE), ISSN : 0975-3397 Vol. 5 No. 07.

[14] Jackson D. J.m "Computer Vision \& Digital Image Processing: Edge Linking and Boundary Detection". https://www.google.iq/?gfe_rd=cr\&ei=NNgzWYS5NrPb 8Afb0g8\&gws_rd=ssl\#q=Computer+V ision+\%26+Digital+Image+Processing+Edge+Linking+a nd+Boundary+Detection

[15] .Jia Li, Lei Yao, Ella H., James Z. W., 2012, "Rhythmic Brushstrokes Distinguish Van Gogh From His Contemporaries: Findings Via Automated Brushstroke" IEEE transactions on pattern analysis and machine intelligence Journal, Volume 34, Issue 6, June 2012, Pages 1159-1176.

[16] Joshi A. D. Late G. N. Sapkal , 2015, " A Novel Approach for Color Image Feature Extraction using Swarm Intelligence", International Journal of Computer Applications (0975 - 8887) Volume 111 No 7, 2015

[17] 22Mallick S., 2015, Computer Vision Resources. http://www.learnopencv.com

[18] 1Price S., 1996, "Edges: The Canny Edge Detector",July4,1996.http://homepages.inf.ed.ac.uk/rbf/C Vonline/LOCAL_COPIES/MARBLE/low/edges/canny.h tm

[19] Ravneet S. Arora. Ahmed E., 2013, "Towards Automated Classification of Fine-art Painting Style: a Comparative Study", 21st International Conference on Pattern Recognition. , 11-15 Nov. 2012 , Date Added to IEEE Xplore: 14 February 2013.

[20] Reza A.,2014," Empirical Study of Artificial Fish Swarm Algorithm" International Journal of Computing, Communications and Networking,Volume 3, No.1, January - March 2014, ISSN 2319-2720 Available Online at http://warse.org/pdfs/2014/ijccn013120143.pdf 
[21] Ricardo S. C.b, João P. C.a, Fernando De la T., Alexandre B., Gustavo C., 2011, "Time and Order Estimation of Paintings Based on Visual Features and Expert Priors", Proceedings of the SPIE electronic imaging of art ii(2011), Computer vision and image analysis , Volume 7869, id. 78690G (2011). (SPIE Homepage).

[22] Solberg A. .INF $4300-$ Hough transform www.scribd.com/document/325241480/Hough-transform

[23] Sivaramakrishnan R., Arun Ch., 2013," Connected Components Labeling and Extraction Based Interphase Removal from Chromosome Images", International Journal of Bio-Science and Bio- Technology Vol. 5, No. 1, February, 2013.

[24] Siwei L., Daniel R., and Hany F., 2014,"A Digital Technique for Art Authentication"

[25] Veronica A. C. Baterina, Carlos M. Oppus , 2010, "Ant Colony Optimization for Image Edge Detection", Proceeding ISPRA'10 Proceedings of the 9th WSEAS international conference on Signal processing, robotics and automation, page 220-225, World Scientific and Engineering Academy and Society (WSEAS) Stevens Point, Wisconsin, USA (O2010

[26] Yang W., Wei Zh. Hongxing Li., 2014, "Application of artificial fish swarm algorithm in image registration", Computer Modelling \& New Technologies, 2014 18(12B) 510-516

[27] .Zhehuang H. Yidong Ch., 2005" An Improved Artificial Fish Swarm Algorithm based on Hybrid Behavior Selection ", International Journal of Control and Automation Vol.6, No.5 (2013), pp.103-116, ISSN 2005-4297 IJCA Copyright (C) 2013 SERSC http://dx.doi.org/10.14257/ijca.2013.6.5.10

[28] Https://www.mathworks.com/help/images/ref/bwmorph. html

[29] https://www.mathworks.com/matlabcentral/newsreader/v iew_thread/65770

[30] https://en.wikipedia.org/wiki/Canny_edge_detector 